
\title{
R Reserarch Suare \\ Comparative Analysis of Some Schell-Model Beams Propagating Through Turbulent Atmosphere
}

\section{Salma Chib}

Chouaïb Doukkali University

\section{Latifa Dalil-Essakali}

Chouaïb Doukkali University

Abdelmajid Belafhal ( $\square$ belafhal@gmail.com )

Chouaïb Doukkali University https://orcid.org/0000-0003-2735-3108

\section{Research Article}

Keywords: Schell-model beams, Turbulent atmosphere, Huygens-Fresnel diffraction integral, Spectral density.

Posted Date: October 19th, 2021

DOl: https://doi.org/10.21203/rs.3.rs-943353/v1

License: (9) This work is licensed under a Creative Commons Attribution 4.0 International License. Read Full License

Version of Record: A version of this preprint was published at Optical and Quantum Electronics on February 19th, 2022. See the published version at https://doi.org/10.1007/s11082-022-03571-5. 


\title{
Comparative analysis of some Schell-model beams propagating through turbulent atmosphere
}

\author{
S. Chib, L. Dalil-Essakali, A. Belafhal* \\ Laboratory LPNAMME, Laser Physics Group, \\ Department of Physics, Faculty of Sciences, Chouaïb Doukkali University, \\ P. B 20, 24000 El Jadida, Morocco \\ * Corresponding author. E-mail: belafhal@gmail.com
}

\begin{abstract}
In this paper, we investigate a comparative analysis of some Generalized LaguerreGaussian Schell-model beams through a paraxial ABCD optical system in a turbulent atmosphere. Based on the extended Huygens-Fresnel diffraction integral, analytical expressions for the spectral density in the receiver plane of the studied beams are derived in detail. By studying the effects of the source coherence parameters and the atmospheric turbulence strength, the numerical results indicate that the profile of these Schell-model beams takes different shapes during their propagation.
\end{abstract}

Keywords: Schell-model beams; Turbulent atmosphere; Huygens-Fresnel diffraction integral; Spectral density.

\section{Introduction}

In recent years, the propagation of laser beams in a turbulent atmosphere has attracted much attention from researchers [1-7] due to their large applications such as in free space communications [8], active optical imaging [9], and remote sensing [10]. Furthermore, the specific nature of partially coherent laser beams has led to their extensive study in turbulent atmosphere by many authors [11-21]. Lu et al. [17] have interested to the change of degree of coherence of partially coherent Gaussian Schell-model beam during its propagation in a turbulent atmosphere. Zhou and Chu [19] have examined the comportment of partially coherent cosine-Gaussian beam in a turbulent atmosphere through the study of the average intensity. The effect of the atmospheric turbulence and the input beam parameters on the beam width and the average intensity of partially coherent Hermite-cosh-Gaussian beams traveling through atmospheric turbulence has been studied by Yang et al. [20], and recently, Chib et al. [21] have investigated the propagation characteristics of partially coherent Generalized flattened Hermite-cosh-Gaussian beam in a turbulent atmosphere. 
On the other hand, Gori and Santarsiero [22] have presented the sufficient condition of the cross-spectral density (CSD) to be physically realizable. Thereby, considerable analytical models for CSD function of random sources generating flat and ring-shaped intensity profiles in the far field have been introduced such as: multi-Gaussian Schell-model (MGSM) sources, Laguerre-Gaussian Schell-model (LGSM) and Bessel-Gaussian Schell-model (BGSM) sources $[23,24]$. These models have been the subject of different studies; for example, Cang et al. [25] have examined the evolution of the average spectral density, the spectral degree of coherence and the beam width of MGSM beams traveling through atmospheric turbulence. In another work, Cang et al. [26] have reported the propagation characteristics of LGSM and BGSM beams in a turbulent atmosphere. In 2014, Chen et al. [27] have derived the analytical expression for the CSD and the second-order moments of the Wigner distribution function of LGSM beam in a turbulent atmosphere in order to study the statistical properties of this laser beam.

The main objective of the current paper is to study the comportment of the Standard and Elegant Generalized Laguerre-Gaussian Schell-model (GLGSM) beams propagating through a turbulent atmosphere by developing analytically the spectral density, which to our best knowledge, has not evaluated previously. The remainder of the paper is organized as follows: Section 2 presents a general theoretical formulation for the spectral density of Schellmodel beams through a paraxial ABCD optical system in atmospheric turbulence. In Section 3, we derive the analytical expressions of the spectral density for the Gaussian Schell-model, Standard and Elegant Generalized Laguerre-Gaussian Schell-model beams in atmospheric turbulence. Discussions and comparative analysis for the spectral density of the studied Shellmodel beams in the considered medium are exposed detailly in Section 4. A last Section outlines a general conclusion.

\section{Theoretical Formulation}

Based on the extended Huygens-Fresnel integral, the CSD at $z$ plane of some Schellmodel beams in atmospheric turbulence will be investigated. The considered beams in this study are: Gaussian Schell-model (GSM) and GLGSM beams.

Within the paraxial approximation, the corresponding CSD of the collimated beam can be written as

$$
W^{(0)}\left(\mathbf{r}_{1}, \mathbf{r}_{2} ; \omega\right)=\exp \left(-\frac{\mathbf{r}_{1}^{2}+\mathbf{r}_{2}^{2}}{4 \sigma_{s}^{2}}\right) \mu^{(0)}\left(\mathbf{r}_{2}-\mathbf{r}_{1} ; \omega\right) \text {. }
$$


In the above expression, $\mathbf{r}_{1}$ and $\mathbf{r}_{2}$ are two-dimensional position vectors, $\omega$ is the angular frequency, $\sigma_{s}$ is the rms width of the source and $\mu^{(0)}$ is the spectral degree of coherence of the source, which takes a specific form for each beams family.

By applying the extended Huygens-Fresnel integral, the propagation of partially coherent beams through a paraxial $\mathrm{ABCD}$ optical system in a turbulent atmosphere can be written as $[19,28,29]$

$$
\begin{aligned}
& W\left(\boldsymbol{\rho}_{1}, \boldsymbol{\rho}_{2}, z ; \omega\right)=\frac{1}{\lambda^{2} B^{2}} \iiint \int W^{(0)}\left(\mathbf{r}_{1}, \mathbf{r}_{2} ; \omega\right) \exp \left[-\frac{i k}{2 F_{0}}\left(\mathbf{r}_{2}^{2}-\mathbf{r}_{1}^{2}\right)\right] \\
& \quad \times \exp \left\{-\frac{i k}{2 B}\left[\left(A \mathbf{r}_{1}^{2}-2 \mathbf{r}_{1} \cdot \boldsymbol{\rho}_{1}+D \boldsymbol{\rho}_{1}^{2}\right)-\left(A \mathbf{r}_{2}^{2}-2 \mathbf{r}_{2} \cdot \mathbf{\rho}_{2}+D \boldsymbol{\rho}_{2}^{2}\right)\right]\right\} \exp \left[-D_{w}\left(\mathbf{r}_{1}-\mathbf{r}_{2}\right) / 2\right] d^{2} \mathbf{r}_{1} d^{2} \mathbf{r}_{2},
\end{aligned}
$$

where $\boldsymbol{\rho}_{\mathbf{1}}=\left(\rho_{1 x}, \rho_{1 y}\right), \boldsymbol{\rho}_{2}=\left(\rho_{2 x}, \rho_{2 y}\right)$ are two arbitrary transverse position vectors at the receive plane, $\mathrm{A}, \mathrm{B}$, and $\mathrm{D}$ are the transfer matrix elements and $d^{2} \mathbf{r}_{1} d^{2} \mathbf{r}_{2}=d r_{1 x} d r_{1 y} d r_{2 x} d r_{2 y}$. By setting $\boldsymbol{\rho}_{1}=\boldsymbol{\rho}_{2}=\boldsymbol{\rho}$ in Eq. (2-a) we obtain the expression for the spectral density ( i.e. the optical intensity at frequency $\omega$ ) of the beam field as follows

$$
\begin{aligned}
S(\boldsymbol{\rho}, z ; \omega) & =\frac{1}{\lambda^{2} B^{2}} \iiint \int W^{(0)}\left(\mathbf{r}_{1}, \mathbf{r}_{2} ; \omega\right) \exp \left[-\frac{i k}{2 F_{0}}\left(\mathbf{r}_{2}^{2}-\mathbf{r}_{1}^{2}\right)\right] \\
& \times \exp \left[\frac{i k}{2 B}\left(A \mathbf{r}_{2}^{2}-A \mathbf{r}_{1}^{2}-2 \mathbf{r}_{2} \boldsymbol{\rho}+2 \mathbf{r}_{1} \mathbf{\rho}\right)\right] \exp \left[-D_{w}\left(\mathbf{r}_{1}-\mathbf{r}_{2}\right) / 2\right] d^{2} \mathbf{r}_{1} d^{2} \mathbf{r}_{2},
\end{aligned}
$$

where $z$ is the propagation distance, $k=2 \pi / \lambda$ is the wave number with $\lambda$ is the wavelength and $F_{0}$ is the wave-front radius of curvature of the input field. $D_{w}$ is the generalized wavestructure function given, in the Kolmogorov turbulence spectrum, by [28]

$D_{w}\left(\mathbf{r}_{1}-\mathbf{r}_{2}\right)=2\left[\frac{\left|r_{1}-r_{2}\right|}{\sigma_{0}}\right]^{5 / 3}$,

with $\sigma_{0}=\left(0.545 C_{n}^{2} k^{2} z\right)^{-3 / 5}$ is the spherical wave lateral coherence length of the optical system and $C_{n}^{2}$ represents the refraction index structure constant.

By substituting Eqs. (1) and (3) into Eq. (2-b), and by introducing the change of variables: $\mathbf{s}_{1}=\frac{\left(\mathbf{r}_{1}+\mathbf{r}_{2}\right)}{2}$ and $\mathbf{s}_{2}=\mathbf{r}_{2}-\mathbf{r}_{1}$, the following expression for the spectral density of the beam, at a particular frequency $\omega$, is obtained 


$$
\begin{aligned}
S(\boldsymbol{\rho}, z ; \omega)= & \frac{1}{\lambda^{2} B^{2}} \iiint \int \exp \left(-\frac{2 \mathbf{s}_{\mathbf{1}}^{2}+\frac{\mathbf{s}_{\mathbf{2}}^{2}}{2}}{4 \sigma_{s}^{2}}\right) \mu^{(0)}\left(\mathbf{s}_{2} ; \omega\right) \exp \left(-\frac{i k}{F_{0}} \mathbf{s}_{\mathbf{1}} \mathbf{s}_{\mathbf{2}}\right) \\
& \times \exp \left(\frac{i k}{B} \mathbf{s}_{\mathbf{1}} \mathbf{s}_{\mathbf{2}}-\frac{i k \rho}{B} \mathbf{s}_{\mathbf{2}}\right) \exp \left[-\frac{D_{w}\left(\mathbf{s}_{\mathbf{2}}\right)}{2}\right] d^{2} \mathbf{s}_{\mathbf{1}} d^{2} \mathbf{s}_{\mathbf{2}} .
\end{aligned}
$$

After some algebraic calculations and integral operation, Eq. (4) can be written as

$$
S(\boldsymbol{\rho}, z ; \omega)=\frac{4 \pi^{2} \sigma_{s}^{2}}{\lambda^{2} B^{2}} \int_{0}^{+\infty} \exp \left(-\tilde{\eta} \mathbf{s}_{2}^{2}\right) \exp \left[-\left(\frac{\left|\mathbf{s}_{\mathbf{2}}\right|}{\sigma_{0}}\right)^{5 / 3}\right] \mu^{(0)}\left(\mathbf{s}_{2} ; \omega\right) J_{0}\left(\frac{k \rho \mathbf{s}_{\mathbf{2}}}{B}\right) \mathbf{s}_{\mathbf{2}} d \mathbf{s}_{\mathbf{2}},
$$

where

$$
\tilde{\eta}=\frac{1}{8 \sigma_{s}^{2}}+\frac{k^{2} \sigma_{s}^{2}}{2 B^{2}}\left(A-\frac{B}{F_{0}}\right)^{2} .
$$

Note that from Eq. (3), the generalized wave-structure function is a power somewhat less than two, and that the exact value $5 / 3$ is not crucial. Therefore, Wandzura [30] has proposed a quadratic approximation of $D_{w}$ by expanding the $5 / 3$ power as

$x^{2-\varepsilon}=x^{2}\left(1-\varepsilon \ln x+1 / 2 \varepsilon^{2} \ln ^{2} x-\ldots\right)$,

with $\varepsilon=\frac{1}{3}$.

Applying this last equation to the factor exp $\left[-\left(\frac{\mathbf{s}_{\mathbf{2}}}{\sigma_{0}}\right)^{5 / 3}\right]$, one obtains

$\exp \left[-\left(\frac{\mathbf{s}_{\mathbf{2}}}{\sigma_{0}}\right)^{5 / 3}\right]=\exp \left(-\frac{\mathbf{s}_{\mathbf{2}}^{2}}{\sigma_{0}^{2}}\right)+\frac{\mathbf{s}_{\mathbf{2}}^{2}}{3 \sigma_{0}^{2}} \ln \left(\frac{\mathbf{s}_{\mathbf{2}}}{\sigma_{0}}\right) \exp \left(-\frac{\mathbf{s}_{\mathbf{2}}^{2}}{\sigma_{0}^{2}}\right)+\ldots$

By the use of Eq. (8), Eq. (5) can be further expressed as

$$
S(\boldsymbol{\rho}, z ; \omega)=\frac{4 \pi^{2} \sigma_{s}^{2}}{\lambda^{2} B^{2}}\left(I(\boldsymbol{\rho}, z ; \omega)+\frac{J(\boldsymbol{\rho}, z ; \omega)}{3 \sigma_{0}^{2}}\right),
$$

where

$$
I(\boldsymbol{\rho}, z ; \omega)=\int_{0}^{+\infty} \exp \left(-\eta^{\prime} t^{2}\right) \mu^{(0)}(\mathbf{t} ; \omega) J_{0}\left(\frac{k \rho t}{B}\right) t d t,
$$

and

$$
J(\boldsymbol{\rho}, z ; \omega)=\int_{0}^{+\infty} t^{3} \exp \left(-\eta^{\prime} t^{2}\right) \mu^{(0)}(\mathbf{t} ; \omega) J_{0}\left(\frac{k \rho t}{B}\right) \ln \left(\frac{t}{\sigma_{0}}\right) d t,
$$


with

$\eta^{\prime}=\tilde{\eta}+\frac{1}{\sigma_{0}^{2}}$

and $J_{0}$ is the Bessel function of order zero.

To evaluate the spectral density $S(\boldsymbol{\rho}, z ; \omega)$ at a particular frequency, the two integrals $I(\boldsymbol{\rho}, z ; \omega)$ and $J(\boldsymbol{\rho}, z ; \omega)$ for each beam will be derived in the below Section. Note that Eq. (9) is our first general main result in the present work.

\section{Spectral density of some Schell-model beams}

In the following, we will develop the analytical expression of the spectral density for the Gaussian Schell-model beams and GLGSM beams.

\subsection{Gaussian Schell-model beams}

The spectral degree of coherence of the source for the Gaussian Schell-model (GSM) beams is given as

$$
\mu_{G S M}^{(0)}\left(\mathbf{r}_{2}-\mathbf{r}_{1} ; \omega\right)=\mu_{G}^{(0)}\left(\mathbf{r}_{2}-\mathbf{r}_{1} ; \omega\right)=\exp \left(-\frac{\left|\mathbf{r}_{2}-\mathbf{r}_{1}\right|^{2}}{\delta^{2}}\right),
$$

where $\delta$ is a coherence width.

By substituting this last expression in Eqs. (9), the following integrals are yielded

$$
I_{G S M}(\boldsymbol{\rho}, z ; \omega)=\int_{0}^{+\infty} \exp \left(-\eta t^{2}\right) J_{0}(\mu t) t d t,
$$

and

$$
J_{G S M}(\boldsymbol{\rho}, z ; \omega)=\int_{0}^{+\infty} t^{3} \exp \left(-\eta t^{2}\right) J_{0}(\mu t) \ln \left(\frac{t}{\sigma_{0}}\right) d t,
$$

with

$\eta=\eta^{\prime}+\frac{1}{\delta^{2}}$

and

$\mu=\frac{k \rho}{B}$.

The evaluation of Eq. (11) is realizable with the help of the identity [31] 


$$
\int_{0}^{+\infty} t^{s} \exp \left(-\eta t^{2}\right) J_{v}(\beta t) d t=\frac{\beta^{v} \Gamma\left(\frac{s+v+1}{2}\right)}{2^{v+1} \eta^{\frac{s+v+1}{2}} v !} F_{1}\left(\frac{s+v+1}{2} ; v+1 ;-\frac{\beta^{2}}{4 \eta}\right),
$$

with $\operatorname{Re}(\eta)>0, \operatorname{Re}(s+v)>-1, J_{v}$ is the Bessel function of the first kind and order $v, \Gamma$ is the gamma function and ${ }_{1} F_{1}$ is the Kummer's series of the confluent hypergeometric series.

So, one obtains

$$
I_{G S M}(\boldsymbol{\rho}, z ; \omega)=\frac{\exp \left(-\mu^{2} / 4 \eta\right)}{2 \eta} .
$$

To evaluate Eq. (12), we recall the following identities [31]

$$
J_{0}(\mu t)=\sum_{h=0}^{+\infty} \frac{(-1)^{h}}{(h !)^{2}}\left(\frac{\mu t}{2}\right)^{2 h}
$$

and

$$
\int_{0}^{+\infty} t^{s} \exp \left(-a t^{2}\right) \ln (b t) d t=\frac{1}{4 \sqrt{a^{(s+1)}}} \Gamma\left(\frac{s+1}{2}\right)\left[\ln \left(\frac{b^{2}}{a}\right)+\psi\left(\frac{s+1}{2}\right)\right],
$$

to obtain

$$
J_{G S M}(\boldsymbol{\rho}, z ; \omega)=\frac{1}{4 \eta^{2}} \sum_{j=0}^{+\infty}(j+1) \frac{\left(-\mu^{2} / 4 \eta\right)^{j}}{j !}\left[\ln \left(\frac{1}{\eta \sigma_{0}^{2}}\right)+\psi(j+2)\right],
$$

where $\psi$ is the digamma function defined by $\psi(x)=\frac{\Gamma^{\prime}(x)}{\Gamma(x)}$.

By using the identity [32]

$$
\begin{aligned}
& \sum_{j=0}^{+\infty} \frac{(j+1)}{j !}(-z)^{j}\left[\ln \left(\frac{1}{\eta \sigma_{0}^{2}}\right)+\psi(j+2)\right] \\
& \quad=\exp (-z)\left\{[2-\exp (z)]+(z-1)\left[\operatorname{chi}(z)+\operatorname{shi}(z)-\ln \left(\frac{1}{\eta \sigma_{0}^{2}} z\right)\right]\right\},
\end{aligned}
$$

where $c h i$ and $s h i$ are the hyperbolic cosine and sine integral functions, with $z=\frac{\mu^{2}}{4 \eta}$, Eq. (17) can be written as

$$
J_{G S M}(\boldsymbol{\rho}, z ; \omega)=\frac{1}{4 \eta^{2}} \exp \left(-\frac{\mu^{2}}{4 \eta}\right)\left\{\left[2-\exp \left(\frac{\mu^{2}}{4 \eta}\right)\right]+\left(\frac{\mu^{2}}{4 \eta}-1\right)\left[\operatorname{chi}\left(\frac{\mu^{2}}{4 \eta}\right)+\operatorname{shi}\left(\frac{\mu^{2}}{4 \eta}\right)-\ln \left(\frac{\mu^{2}}{4 \eta^{2} \sigma_{0}^{2}}\right)\right]\right\} \text {. }
$$

Finally, Eq. (9) can be rearranged, in the case of GSM beams, as 


$$
\begin{aligned}
& S_{G S M}(\boldsymbol{\rho}, z ; \omega)=\frac{2 \pi^{2} \sigma_{s}^{2}}{\lambda^{2} B^{2}} \frac{\exp \left(-\mu^{2} / 4 \eta\right)}{\eta} \\
& \times\left\{1+\frac{1}{6 \eta \sigma_{0}^{2}}\left\{\left(2-\exp \left(\frac{\mu^{2}}{4 \eta}\right)\right)+\left(\frac{\mu^{2}}{4 \eta}-1\right)\left[\operatorname{chi}\left(\frac{\mu^{2}}{4 \eta}\right)+\operatorname{shi}\left(\frac{\mu^{2}}{4 \eta}\right)-\ln \left(\frac{\mu^{2}}{4 \eta^{2} \sigma_{0}^{2}}\right)\right]\right\}\right\} .
\end{aligned}
$$

The result obtained in Eq. (20) is our second main finding of the spectral density of GSM beams in a turbulent atmosphere. In our best knowledge, this closed form is not published yet in the literature.

For the axial spectral density (i.e. $\boldsymbol{\rho}=\overrightarrow{0}$ ), Eq. (19) becomes

$$
J_{G S M}(\overrightarrow{0}, z ; \omega)=\frac{1}{4 \eta^{2}}\left[\ln \left(\frac{1}{\eta \sigma_{0}^{2}}\right)+1-\gamma\right],
$$

with $\gamma$ is the Euler's constant, and Eq. (20) can be written, in this case, as

$$
S_{G S M}(\overrightarrow{0}, z ; \omega)=\frac{2 \pi^{2} \sigma_{s}^{2}}{\lambda^{2} B^{2} \eta}\left\{1+\frac{1}{6 \eta \sigma_{0}^{2}}\left[\ln \left(\frac{1}{\eta \sigma_{0}^{2}}\right)+1-\gamma\right]\right\} \text {. }
$$

Eq. (22) describes the approximation expression of the axial spectral density of GSM beams in a turbulent atmosphere.

\subsection{Generalized Laguerre-Gaussian Schell-model beams}

The spectral degree of coherence of the source for the Generalized Laguerre-Gaussian Schell-model (GLGSM) beams is expressed by

$$
\mu_{G L G S M}^{(0)}\left(\mathbf{r}_{2}-\mathbf{r}_{1} ; \omega\right)=\mu_{G}^{(0)}\left(\mathbf{r}_{2}-\mathbf{r}_{1}\right) L_{l}^{(\alpha)}\left(\frac{q^{2}\left|\mathbf{r}_{2}-\mathbf{r}_{1}\right|^{2}}{\delta^{2}}\right),
$$

where $L_{l}^{(\alpha)}$ is the generalized Laguerre polynomial, $\delta$ is a coherence width and $q$ is a parameter, which defines the different types of Laguerre-Gaussian beams. For $q=\sqrt{2}$, Eq. (23) presents the spectral degree of coherence of the source for the Standard GLGSM beams and for $q=1$ it reduces to the spectral degree of coherence of the source for the Elegant GLGSM beams.

For GLGSM beams, Eqs. (9-a and 9-b) take the following forms

$$
I_{G L G S M}^{(l, \alpha)}(\boldsymbol{\rho}, z ; \omega)=\int_{0}^{+\infty} \exp \left(-\eta t^{2}\right) L_{l}^{(\alpha)}\left(\frac{q^{2} t^{2}}{\delta^{2}}\right) J_{0}(\mu t) t d t
$$

and 


$$
J_{G L G S M}^{(l, \alpha)}(\boldsymbol{\rho}, z ; \omega)=\int_{0}^{+\infty} t^{3} \exp \left(-\eta t^{2}\right) L_{l}^{(\alpha)}\left(\frac{q^{2} t^{2}}{\delta^{2}}\right) J_{0}(\mu t) \ln \left(\frac{t}{\sigma_{0}}\right) d t,
$$

where

$$
\eta=\eta^{\prime}+\frac{1}{\delta^{2}} \text {, with } \eta^{\prime} \text { is given by Eq. (9-c). }
$$

One notes that these last quantities given by Eqs. (24) and (25) depend also on the parameter $\alpha$ of the generalized Laguerre polynomial.

The below integral, established recently [33], is used to compute the quantity $I_{G L G S M}^{(l, \alpha)}(\boldsymbol{\rho}, z ; \omega)$

$$
\begin{aligned}
\int_{0}^{+\infty} x^{2 \gamma+1} & \exp \left(-\sigma x^{2}\right) L_{n}^{(\alpha)}\left(\beta x^{2}\right) J_{0}(\mu x) d x \\
= & \frac{\Gamma(n+\alpha+1)}{2 \sigma^{(\gamma+1)}} \exp \left(-\frac{\mu^{2}}{4 \sigma}\right) \sum_{k=0}^{n} \frac{\Gamma(\gamma+k+1)(-\beta / \sigma)^{k}}{k ! \Gamma(n-k+1) \Gamma(\alpha+k+1)} L_{k+\gamma}\left(\frac{\mu^{2}}{4 \sigma}\right) .
\end{aligned}
$$

Then, Eq. (24) can be written as

$$
I_{G L G S M}^{(l, \alpha)}(\boldsymbol{\rho}, z ; \omega)=\frac{\Gamma(l+\alpha+1)}{2 \eta} \exp \left(-\frac{\mu^{2}}{4 \eta}\right) \sum_{p=0}^{l} \frac{\left(-q^{2} / \eta \delta^{2}\right)^{p}}{\Gamma(l-p+1) \Gamma(\alpha+p+1)} L_{p}\left(\frac{\mu^{2}}{4 \eta}\right),
$$

and if $\alpha=0$, this last equation becomes

$$
I_{L S S M}^{(l, 0)}(\boldsymbol{\rho}, z ; \omega)=\frac{1}{2 \eta}\left(1-\frac{q^{2}}{\delta^{2} \eta}\right)^{l} \exp \left(-\frac{\mu^{2}}{4 \eta}\right) L_{l}\left[\frac{q^{2} \mu^{2}}{4 \delta^{2} \eta\left(\frac{1}{2 \delta^{2}}-\eta\right)}\right] .
$$

By using the below formula [34]

$$
\begin{aligned}
\int_{0}^{+\infty} x^{2 \gamma+1} & \exp \left(-\sigma x^{2}\right) L_{n}^{(\alpha)}\left(\beta x^{2}\right) J_{0}(\mu x) \ln (b x) d x \\
& =\frac{1}{4 \sigma^{(\gamma+1)}} \sum_{m=0}^{n} \frac{\Gamma(n+\alpha+1)(-\beta / \sigma)^{m}}{\Gamma(n-m+1) \Gamma(\alpha+m+1) \mathrm{m} !} \Gamma(\gamma+m+1) A_{m, 0},
\end{aligned}
$$

where

$$
\begin{aligned}
& A_{m, 0}=\left[\ln \left(\frac{b^{2}}{\sigma}\right)+\psi(\gamma+m)\right]{ }_{1} F_{1}\left(\gamma+m+1 ; 1 ;-\mu^{2} / 4 \sigma\right) \\
& +\frac{1}{(\gamma+m)} F_{2: 1 ; 0}^{1: 2 ; 1}\left[\begin{array}{cc}
\gamma+m+1: & \gamma+m, 1 ; 1 ; \\
1,1: & \gamma+m+1 ;-;
\end{array}-\mu^{2} / 4 \sigma,-\mu^{2} / 4 \sigma\right],
\end{aligned}
$$

and $(b>0, \operatorname{Re}(\sigma)>0, \operatorname{Re}(2 \gamma+1)>-1)$, with $F_{2: 1 ; 0}^{1: 2 ; 1}$ is the Kampé de Feriet series [31], we can easily evaluate the integral of Eq. (25) as follows 


$$
J_{G L G S M}^{(l, \alpha)}(\boldsymbol{\rho}, z ; \omega)=\frac{1}{4 \eta^{2}} \sum_{m=0}^{l} \frac{\Gamma(l+\alpha+1)\left(-q^{2} / \delta^{2} \eta\right)^{m}}{\Gamma(l-m+1) \Gamma(\alpha+m+1) m !} \Gamma\left(\chi_{m}\right) A_{m, 0},
$$

where

$$
\chi_{m}=m+2,
$$

and

$$
\begin{aligned}
& A_{m, 0}=\left[\ln \left(\frac{1}{\sigma_{0}^{2} \eta}\right)+\psi\left(\chi_{m}-1\right)\right]{ }_{1} F_{1}\left(\chi_{m} ; 1 ;-\mu^{2} / 4 \eta\right) \\
& -\frac{1}{\left(1-\chi_{m}\right)} F_{2: 1 ; 0}^{1: 2 ; 1}\left[\begin{array}{cc}
\chi_{m}: & \chi_{m}-1,1 ; 1 ; \\
1,1: & \chi_{m} ; \quad-;
\end{array}-\mu^{2} / 4 \eta,-\mu^{2} / 4 \eta\right] \text {. }
\end{aligned}
$$

If $\alpha=0$, Eq. (32) can be expressed as

$$
J_{\text {LGSM }}^{(l, 0)}(\boldsymbol{\rho}, z ; \omega)=\frac{l !}{4 \eta^{2}} \sum_{m=0}^{l} \frac{\left(-q^{2} / \delta^{2} \eta\right)^{m}}{\Gamma(l-m+1)} \frac{(m+1)}{m !} A_{m, 0} .
$$

Finally, the expressions of the spectral density in turbulent atmosphere are

a - for $\alpha \neq 0$

$$
S_{G L G S M}^{(l, \alpha)}(\boldsymbol{\rho}, z ; \omega)=\frac{4 \pi^{2} \sigma_{s}^{2}}{\lambda^{2} B^{2}}\left(I_{G L G S M}^{(l, \alpha)}(\boldsymbol{\rho}, z ; \omega)+\frac{J_{G L G S M}^{(l, \alpha)}(\boldsymbol{\rho}, z ; \omega)}{3 \sigma_{0}^{2}}\right),
$$

where $I_{G L G S M}^{(l, \alpha)}(\boldsymbol{\rho}, z ; \omega)$ and $J_{G L G S M}^{(l, \alpha)}(\boldsymbol{\rho}, z ; \omega)$ are given by Eqs. (28) and (32), respectively,

b - and for $\alpha=0$

$$
S_{L G S M}^{(l, 0)}(\boldsymbol{\rho}, z ; \omega)=\frac{4 \pi^{2} \sigma_{s}^{2}}{\lambda^{2} B^{2}}\left(I_{L G S M}^{(l, 0)}(\boldsymbol{\rho}, z ; \omega)+\frac{J_{L G S M}^{(l, 0)}(\boldsymbol{\rho}, z ; \omega)}{3 \sigma_{0}^{2}}\right),
$$

where $I_{L G S M}^{(l, 0)}(\boldsymbol{\rho}, z ; \omega)$ and $J_{L G S M}^{(l, 0)}(\boldsymbol{\rho}, z ; \omega)$ are given by Eqs. (29) and (33), respectively.

From the above equations, it is interesting to deduce the expressions of the spectral density propagating through a paraxial ABCD optical system in free space without turbulence which are given as

a - for $\alpha \neq 0$

$$
S_{G L G S M}^{(l, \alpha) F S}(\boldsymbol{\rho}, z ; \omega)=\frac{4 \pi^{2} \sigma_{s}^{2}}{\lambda^{2} B^{2}} I_{G L G S M}^{(l, \alpha) F S}(\boldsymbol{\rho}, z ; \omega),
$$

b - and for $\alpha=0$

$$
S_{L G S M}^{(l, 0) F S}(\boldsymbol{\rho}, z ; \omega)=\frac{4 \pi^{2} \sigma_{s}^{2}}{\lambda^{2} B^{2}} I_{L G S M}^{(l, 0) F S}(\boldsymbol{\rho}, z ; \omega),
$$

where 
$I_{G L G S M}^{(l, \alpha) F S}(\boldsymbol{\rho}, z ; \omega)=\frac{\Gamma(l+\alpha+1)}{2 \eta^{F S}} \exp \left(-\frac{\mu^{2}}{4 \eta^{F S}}\right) \sum_{p=0}^{l} \frac{\left(-q^{2} / \eta^{F S} \delta^{2}\right)^{p}}{\Gamma(l-p+1) \Gamma(\alpha+p+1)} L_{p}\left(\frac{\mu^{2}}{4 \eta^{F S}}\right)$,

and

$I_{L G S M}^{(l, 0) F S}(\boldsymbol{\rho}, z ; \omega)=\frac{1}{2 \eta^{F S}}\left(1-\frac{q^{2}}{\delta^{2} \eta^{F S}}\right)^{l} \exp \left(-\frac{\mu^{2}}{4 \eta^{F S}}\right) L_{l}\left[\frac{q^{2} \mu^{2}}{4 \delta^{2} \eta^{F S}\left(\frac{1}{2 \delta^{2}}-\eta^{F S}\right)}\right]$,

with $\eta^{F S}=\tilde{\eta}+\frac{1}{\delta^{2}}$.

\section{Numerical simulations and discussion}

In this Section, the propagation properties of GSM and GLGSM beams in a turbulent atmosphere are numerically illustrated by using the analytical expressions of the spectral density developed in Section 3. For the simulations, the wave-front radius curvature of the beams is considered to tend toward infinity and the rms width of the source is taken as $\sigma_{s}=5 \mathrm{~cm}$.

The influences of the atmospheric turbulence and the incident beams parameters on the spectral density at the receiver plane of the studied beams family are represented in the below figures. In Fig. 1, the normalized spectral density of GSM beams versus $\rho$ is plotted for various values of the turbulent strength $C_{n}^{2}$ at $z=6 \mathrm{~km}$ and $8 \mathrm{~km}$ with $\lambda=1064 \mathrm{~nm}$ and $\delta=4.24 \mathrm{~cm}$.

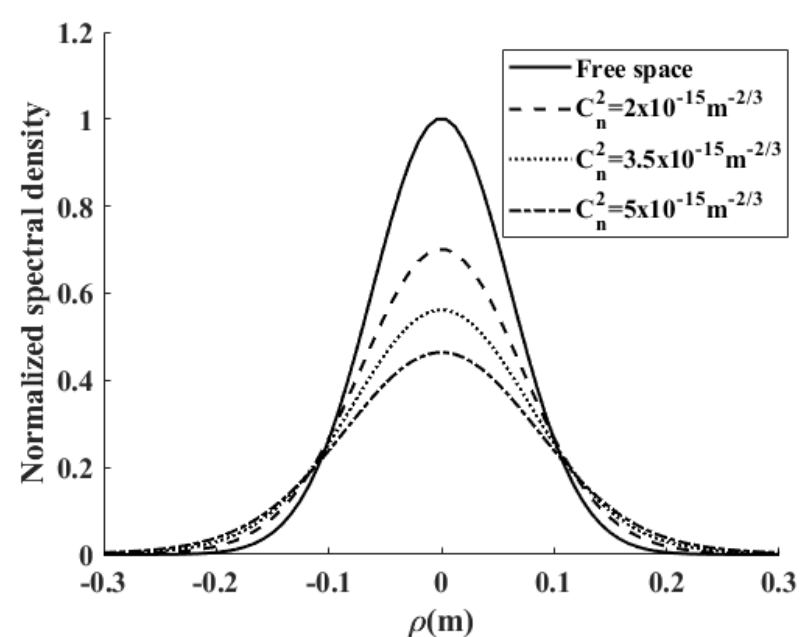

(a)

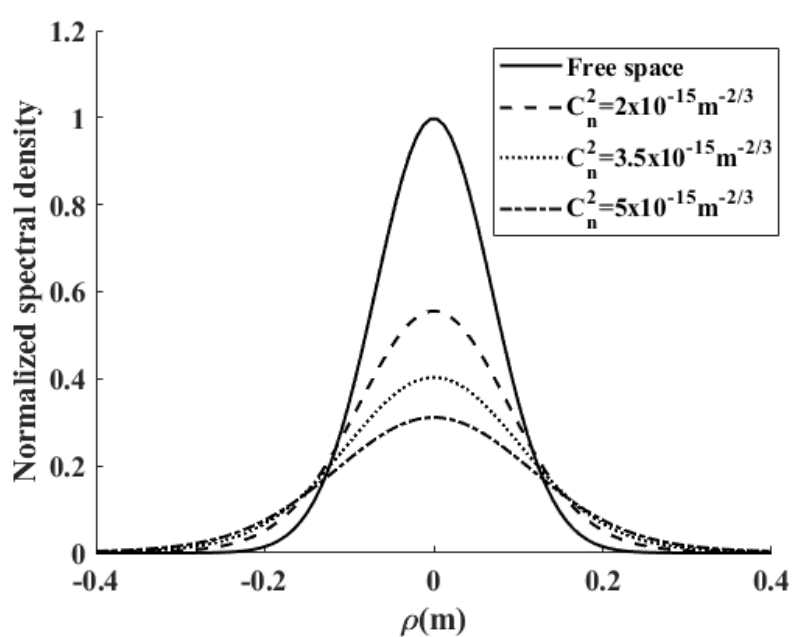

(b)

Figure 1: Normalized spectral density versus $\rho$ of the GSM beams in free space and through a turbulent atmosphere for different values of $C_{n}^{2}$ at (a) $z=6 \mathrm{~km}$ and (b) $z=8 \mathrm{~km}$. 
It is noted from the figure that the maximum of the spectral density of GSM beams weakens when the environment is more turbulent as the propagation distance increases.

Fig. 2 shows the effects of the coherence width $\delta$ and the wavelength $\lambda$ on the behavior of GSM beams upon their propagation through an atmospheric turbulence. The other parameters are set to be $z=6 \mathrm{~km}$ and $C_{n}^{2}=5 \times 10^{-15} \mathrm{~m}^{-2 / 3}$. From this figure, it can be observed that as the coherence width is large as the maximum of the spectral density increases (see Fig. 2(a)). The same comportment is observed by increasing the value of the wavelength of the GSM beams for the values lower than $\lambda=1064 \mathrm{~nm}$, while the maximum of the spectral density starts to decrease when $\lambda>1064 \mathrm{~nm}$ (see Fig. 2(b)).

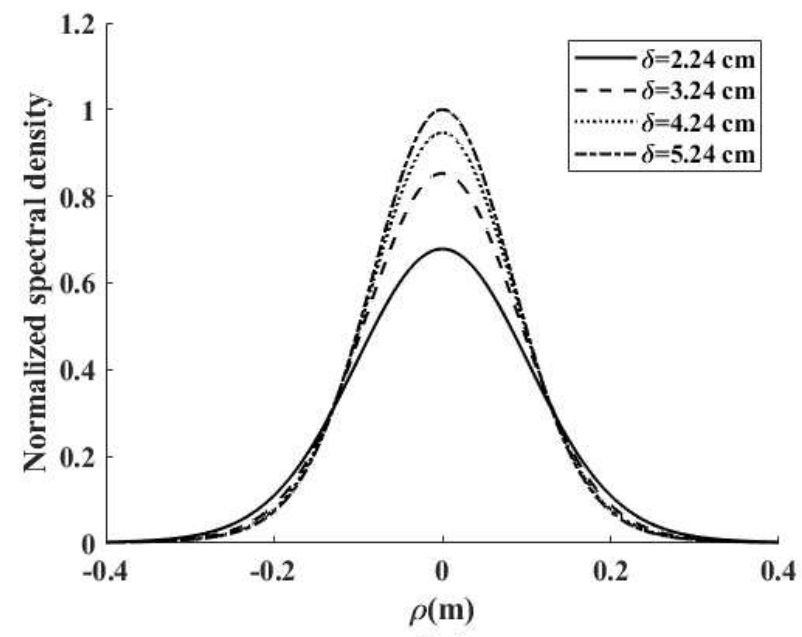

(a)

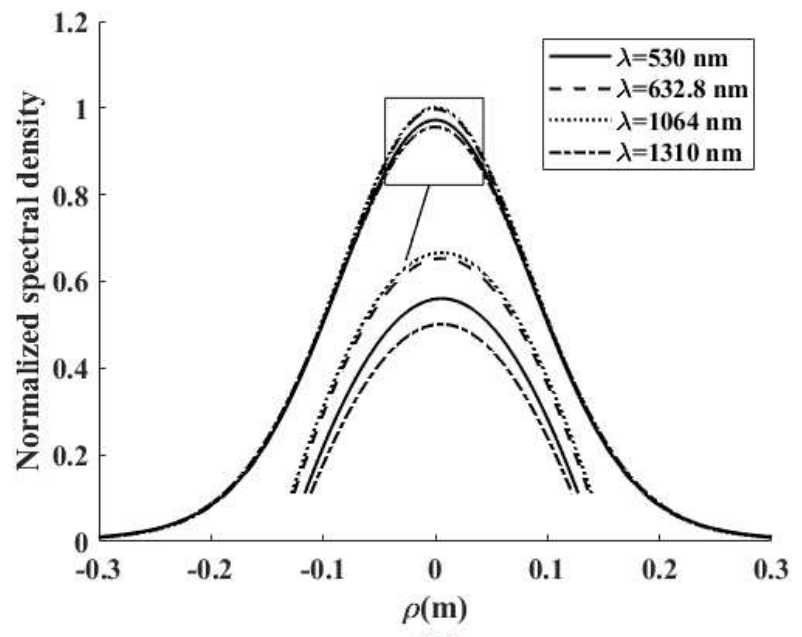

(b)

Figure 2: Normalized spectral density of the GSM beams traveling through a turbulent atmosphere for $z=6 \mathrm{~km}$ and $C_{n}^{2}=5 \times 10^{-15} \mathrm{~m}^{-2 / 3}$, with (a) $\lambda=1064 \mathrm{~nm}$ and various values of $\delta$, (b) $\delta=4.24 \mathrm{~cm}$ and different values of $\lambda$.

We illustrate in Figs. 3 and 4 the normalized spectral density given by Eqs. (34) and (36) versus $\rho$ for various values of the turbulent strength $C_{n}^{2}$ and the beam order $l$ at two values of the propagation distance, with $\lambda=1064 \mathrm{~nm}, \alpha=0.25$ and $\delta=4.24 \mathrm{~cm}$. From Figs. 3(A)-(B), we note that the profiles of Standard GLGSM and Elegant GLGSM beams traveling through atmospheric turbulence and free space display different stages of evolution. It is also observed that the maximum of the spectral density distribution for the two considered beams decreases when the atmosphere is very turbulent. Fig. 3(A) shows that the beam profile of the Standard GLGSM beams evolves from flat-top distribution into Gaussian distribution as the $C_{n}^{2}$ increases for $l=1$. 

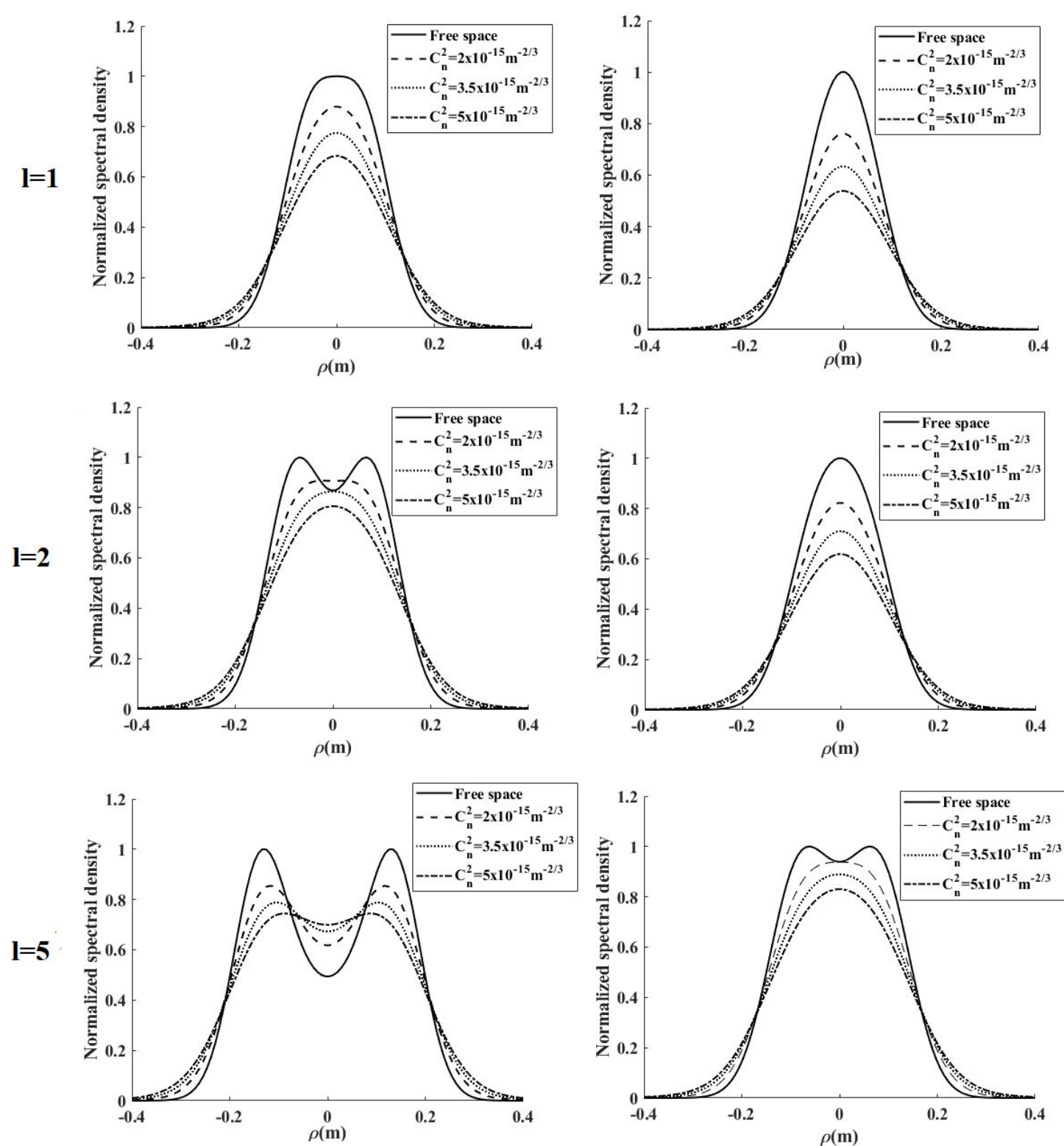

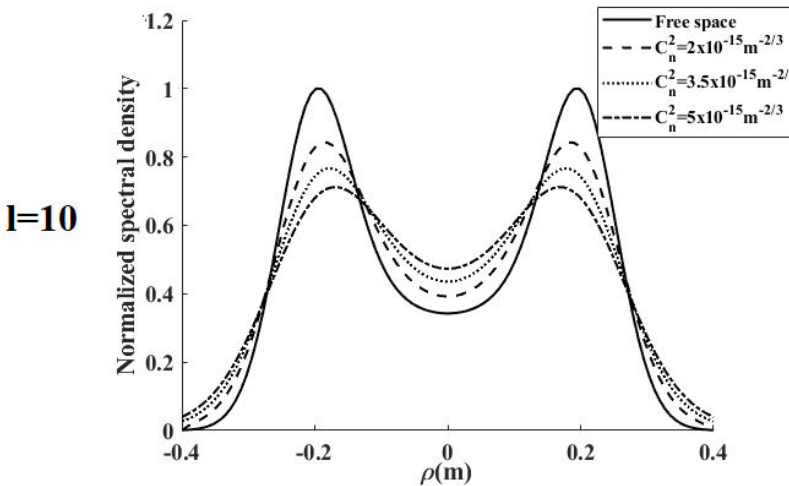

(A)

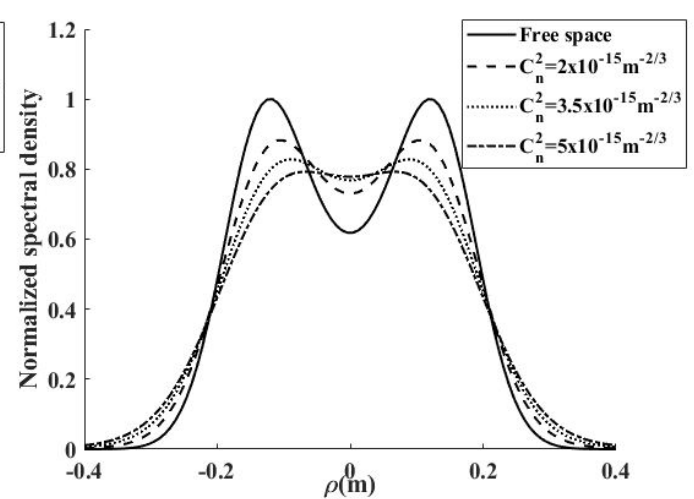

(B)

Figure 3: Normalized spectral density of the GLGSM beams in free space and through a turbulent atmosphere for different values of $C_{n}^{2}$ and of the beam order $l$ with $\alpha=0.25$ and $z=6 \mathrm{~km}$ :

(A) Standard GLGSM and (B) Elegant GLGSM beams. 

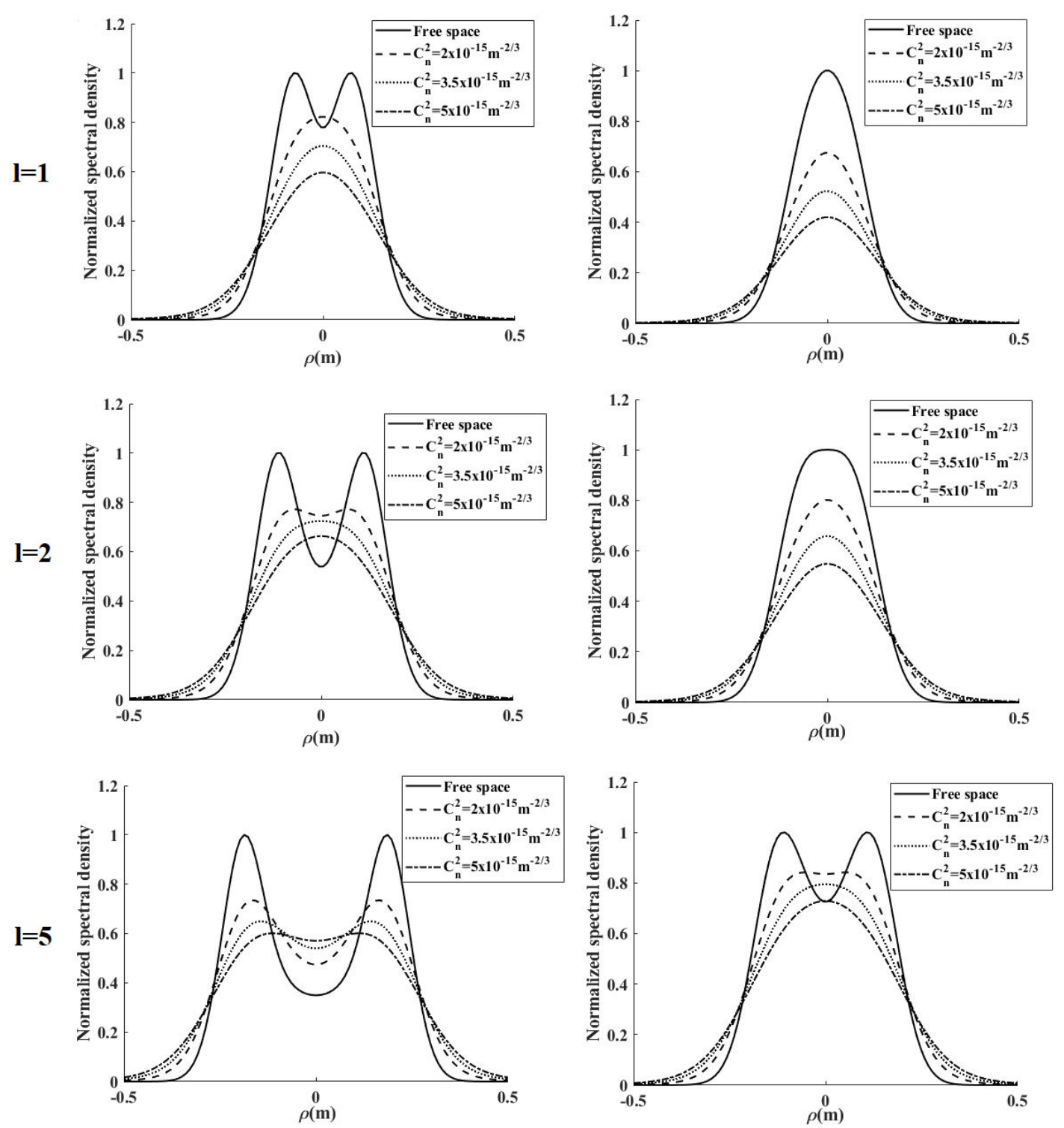

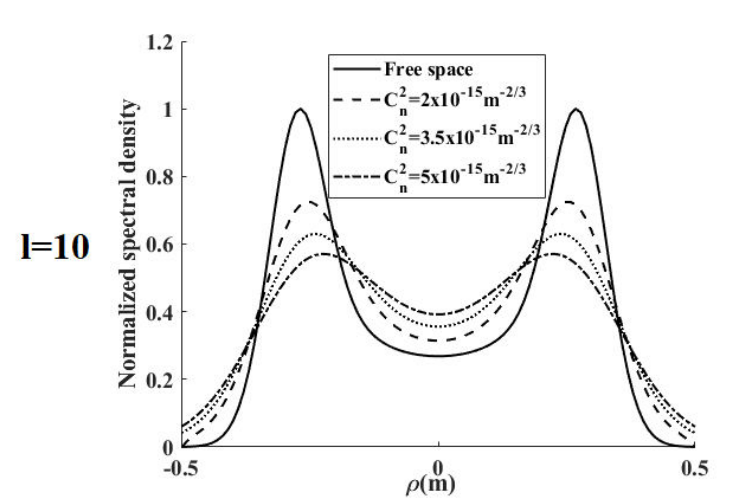

(A)

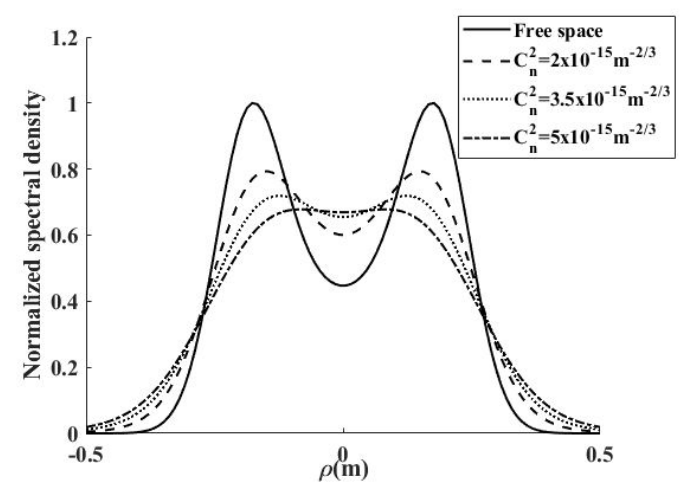

(B)

Figure 4: Normalized spectral density of the GLGSM beams propagating in free space and through a turbulent atmosphere, with $\alpha=0.25$ and $z=8 \mathrm{~km}$ :

(A) Standard GLGSM and (B) Elegant GLGSM beams. 
However, when $l$ is equal to two a ring-shaped beam profile appears in free space without turbulence and transforms into Gaussian profile with the increase of the turbulent strength. Furthermore, we note that for large values of $l$, the beam profile takes ring-shaped form and remains almost unchanged while the hollowness of the ring-shaped beam profile is affected by the variation of the turbulent strength $C_{n}^{2}$.

The behavior evolution of the Elegant GLGSM beams passing through atmospheric turbulence and free space without turbulence differs slightly compared to that one of the Standard GLGSM beams previously treated. From Fig. 3(B), one sees that the beam profile of the Elegant GLGSM beams remains Gaussian distribution for small value of $l$. When $l$ is greater than or equal to five, the Elegant GLGSM beams exhibit another type of profile, gradually passing from a Gaussian profile to a ring-shaped profile. We can also note that the widen of the ring-shaped beam increases with the beam order.

The Standard GLGSM beams distribution becomes a ring-shaped beam profile in free space without turbulence when the propagation distance increases (Fig. 4A). Moreover, the depth of the dip in the spectral density profile increases with the source coherence parameter $l$. While the Elegant GLGSM beams take the same comportment by changing its profile from Gaussian shape, then flat-top shape, and finally turn into ring-shaped when $l$ increases. We can clearly observed in the presence of the turbulence that, with a small value of $l$, the profiles of the Standard GLGSM and the Elegant GLGSM beams keep a Gaussian shape unchanged with the increase of the turbulent strength (see Fig. 4(A) and Fig. 4(B)). When $l=2$, the evolution of the comportment for the Standard GLGSM and the Elegant GLGSM beams propagating in a turbulent atmosphere is similar to that of Fig. 3, with the exception of the depth of the dip is extremely important. From this investigation, we can conclude that this beams family has interesting characteristics, which give them the advantage of being better using in certain applications like trapping and transport of particles.

The effect of the incident beam parameter $\alpha$ on the evolution of the normalized spectral density of GLGSM beams is plotted in Fig. 5. The calculation parameters are: $l=10$, $C_{n}^{2}=5 \times 10^{-15} \mathrm{~m}^{-2 / 3}, \lambda=1064 \mathrm{~nm}, \delta=4.24 \mathrm{~cm}$ and $z=6 \mathrm{~km}$. One sees that the beam profile of Standard GLGSM beams gradually transforms from a central-dip beam shape to a flattopped beam shape and finally to a Gaussian beam shape when the incident beam parameter $\alpha$ gradually increases (see Fig. 5-A). For the Elegant GLGSM beams, its profile undergoes the same process as that of the Standard GLGSM beams while the conversion from a centraldip profile into a Gaussian beam occurs more rapidly. 

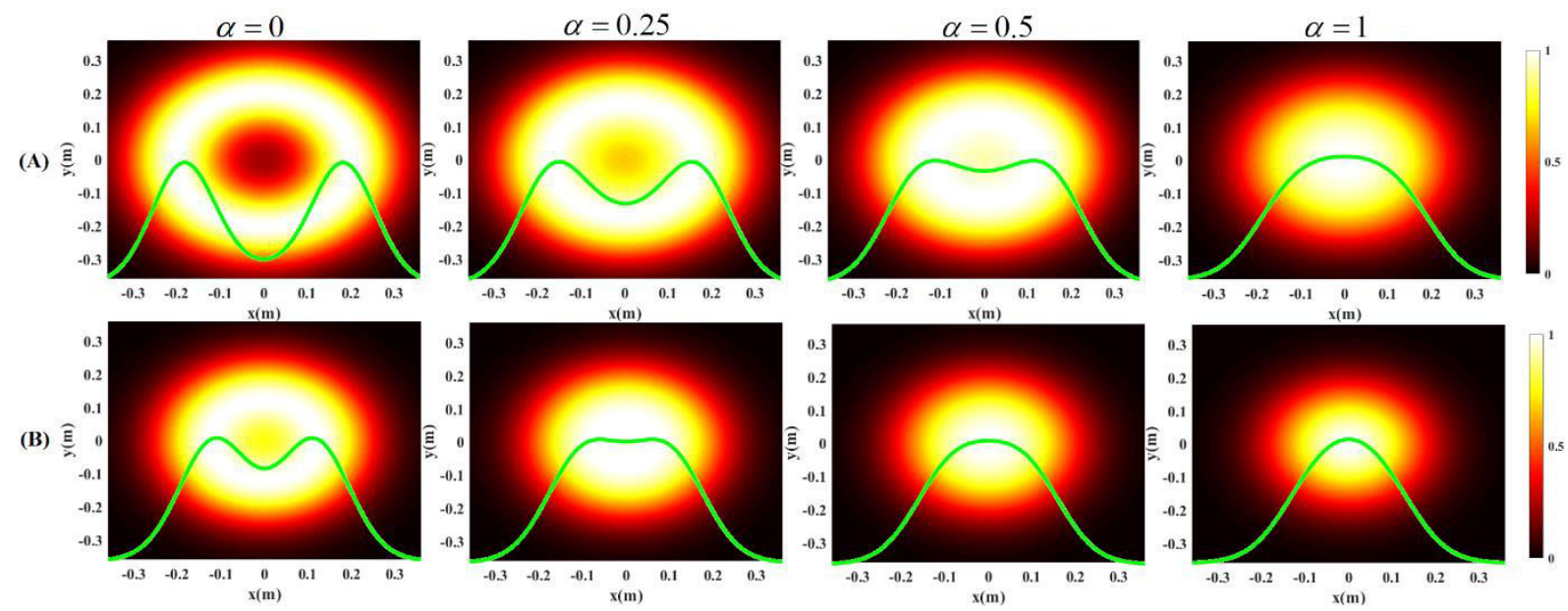

Figure 5: Normalized spectral density of the GLGSM beams propagating in a turbulent atmosphere at $z=6 \mathrm{~km}$, with (A) Standard GLGSM and (B) Elegant GLGSM beams.

We can note that the spectral density of GLGSM beams given by Eq. (34) is reduced to the spectral density of GSM beams for $\alpha=0$ and $l=0$. As it is difficult to prove it analytically, we proceeded to a numerical illustration (see Fig. (6)) to confirm our results.

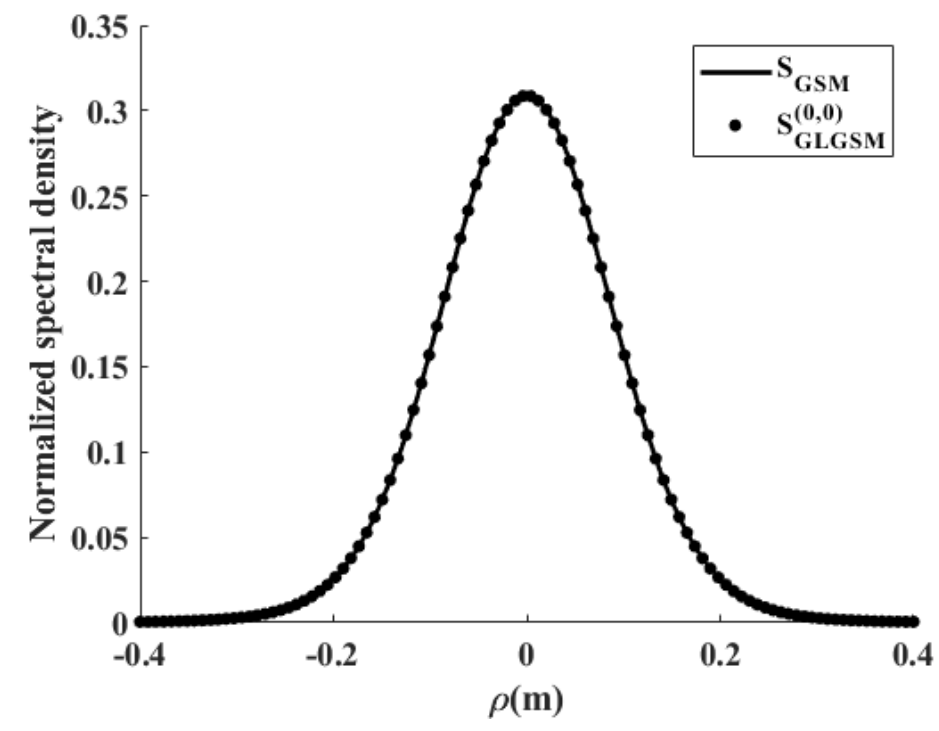

Figure 6: Normalized spectral density of GSM beams $\mathrm{S}_{\mathrm{GSM}}$ and GLGSM beams $\mathrm{S}_{\mathrm{GLGSM}}$ versus $\rho$ in a turbulent atmosphere.

From the plot of this figure, it can be clearly seen that the obtained result from the GLGSM beams is in a good agreement with that in the case of the GSM beams, given by Eq. (20). In our numerical analysis, the following parameters are used: $\alpha=0, l=0, C_{n}^{2}=5 \times 10^{-15} \mathrm{~m}^{-2 / 3}$, $z=6 \mathrm{~km}, \lambda=1064 \mathrm{~nm}$ and $\delta=4.24 \mathrm{~cm}$. 


\section{Conclusion}

In this work, we have derived the analytical expression of the spectral density for GSM and GLGSM beams based on the extended Huygens-Fresnel integral formula in the paraxial approximation. The evolution of the propagation for GSM, Standard and Elegant GLGSM beams through a paraxial ABCD optical system through a turbulent atmosphere is illustrated numerically by varying the input beam parameters and the turbulent strength. From the obtained results, different shapes of beam profile are observed upon the propagation of GLGSM beams through a turbulent atmosphere, which explains that the profile of the studied beams is influenced by the source coherence parameters and the atmospheric turbulence of the environment. These characteristic properties of beams can be useful in practice, such as for optical projection and communications systems. 


\section{References}

[1] A. Kinani, L. Ez-zariy, A. Chafiq, H. Nebdi, A. Belafhal, Effects of atmospheric turbulence on the propagation of Li's flat-topped optical beams, Phys. Chem. News 61 (2011) 24-33.

[2] F. Boufalah, L. Dalil-Essakali, H. Nebdi, A. Belafhal, Effect of turbulent atmosphere on the on-axis average intensity of Pearcey-Gaussian beam, Chinese Physics B 25 (2016) 064208-064213.

[3] F. Saad, A. Belafhal, A theoretical study of the on-axis average intensity of generalized spiraling Bessel beams in a turbulent atmosphere, Opt. Quant. Elect. 49 (2017) 1-12.

[4] A. Belafhal, S. Hennani, L. Ez-zariy, A. Chafiq, M. Khouilid, Propagation of truncated Bessel-modulated Gaussian beams in turbulent atmosphere, Phys. Chem. News 62 (2011) 3643.

[5] L. Ez-zariy, F. Boufalah, L. Dalil-Essakali, A. Belafhal, Effects of a turbulent atmosphere on an apertured Lommel-Gaussian beam, Optik 127 (2016) 11534-11543.

[6] F. Boufalah, L. Dalil-Essakali, L. Ez-zariy, A. Belafhal, Introduction of generalized Bessel-Laguerre-Gaussian beams and its central intensity travelling a turbulent atmosphere, Opt. Quant. Electron. 50 (2018) 305-324.

[7] M. Yaalou, E.M. El Halba, Z. Hricha, A. Belafhal, Propagation characteristics of dark and antidark Gaussian beams in turbulent atmosphere, Opt. Quant. Electron. 51 (2019) 255-264.

[8] S.M. Navidpour, M. Uysal, M. Kavehrad, BER Performance of Free-Space Optical Transmission with Spatial Diversity, IEEE Trans. Wirel. Commun. 6 (2007) 2813-2819.

[9] Z. Hajjarian, M. Kavehrad, J. Fadlullah, Spatially multiplexed multi-input-multi-output optical imaging system in a turbid, turbulent atmosphere, Appl. Opt. 49 (2010) 1528-1538.

[10] L.C Andrews, R.L. Phillips, C.Y. Hopen, Laser beam scintillation with applications, SPIE Press, 2001.

[11] E. Wolf, Introduction to the Theory of Coherence and Polarization of Light, Cambridge University Press, 2007.

[12] O. Korotkova, Model for a partially coherent Gaussian beam in atmospheric turbulence with application in Lasercom, Opt. Eng. 43 (2004) 330-341.

[13] Y. Cai, S. He, Propagation of a partially coherent twisted anisotropic Gaussian Schellmodel beam in a turbulent atmosphere, Appl. Phys. Lett. 89 (2006) 041117-041121.

[14] G. Gbur, O. Korotkova, Angular spectrum representation for the propagation of arbitrary coherent and partially coherent beams through atmospheric turbulence, J. Opt. Soc. Am. 24 (2007) 745-752. 
[15] Y. Cai, Q. Lin, Y. Baykal, H.T. Eyyuboğlu, Off-axis Gaussian Schell-model beam and partially coherent laser array beam in a turbulent atmosphere, Opt. Commun. 278 (2007) 157167.

[16] Y. Yuan, Y. Cai, J. Qu, H.T. Eyyuboğlu, Y. Baykal, O. Korotkova, M $^{2}$-factor of coherent and partially coherent dark hollow beams propagating in turbulent atmosphere, Opt. Express 17 (2009) 17344-17356.

[17] W. Lu, L. Liu, J. Sun, Q. Yang, Y. Zhu, Change in degree of coherence of partially coherent electromagnetic beams propagating through atmospheric turbulence, Opt. Commun. $271(2007) 1-8$.

[18] A. Yang, E. Zhang, X. Ji, B. Lü, Angular spread of partially coherent Hermite-coshGaussian beams propagating through atmospheric turbulence, Opt. Express 16 (2008) 83668380 .

[19] G. Zhou, X. Chu, Propagation of a partially coherent cosine-Gaussian beam through an ABCD optical system in turbulent atmosphere, Opt. Express 17 (2009) 10529-10534.

[20] A. Yang, E. Zhang, X. Ji, B. Lü, Propagation properties of partially coherent Hermitecosh-Gaussian beams through atmospheric turbulence, Opt. Laser Technol. 41 (2009) 714 722.

[21] S. Chib, L. Dalil-Essakali, A. Belafhal, Evolution of the partially coherent Generalized Flattened Hermite-Cosh-Gaussian beam through a turbulent atmosphere, Opt. Quant. Electron. 52 (2020) 484-501.

[22] F. Gori, M. Santarsiero, Devising genuine spatial correlation functions, Opt. Lett. 32 (2007) 3531-3533.

[23] S. Sahin, O. Korotkova, Light sources generating far fields with tunable flat profiles, Opt. Lett. 37 (2012) 2970-2972.

[24] Z. Mei, O. Korotkova, Random sources generating ring-shaped beams, Opt. Lett. 38 (2013) 91-93.

[25] J. Cang, X. Fang, X. Liu, Propagation properties of multi-Gaussian Schell-model beams through ABCD optical systems and in atmospheric turbulence, Opt. Laser Technol. 50 (2013) $65-70$.

[26] J. Cang, P. Xiu, X. Liu, Propagation of Laguerre-Gaussian and Bessel-Gaussian Schellmodel beams through paraxial optical systems in turbulent atmosphere, Opt. Laser Technol. 54 (2013) 35-41.

[27] R. Chen, L. Liu, S. Zhu, G. Wu, F. Wang, Y. Cai, Statistical properties of a LaguerreGaussian Schell-model beam in turbulent atmosphere, Opt. Express 22 (2014) 1871-1883. 
[28] H.T. Yura, S.G. Hanson, Optical beam wave propagation through complex optical systems, J. Opt. Soc. Am. 4 (1987) 1931-1948.

[29] H.T. Yura, S.G. Hanson, Second-order statistics for wave propagation through complex optical systems, J. Opt. Soc. Am. 6 (1989) 564-575.

[30] S.M. Wandzura, Systematic corrections to quadratic approximations for power-law structure functions: the $\delta$ expansion, J. Opt. Soc. Am. 71 (1981) 321-326.

[31] I.S. Gradshteyn, I.M. Ryzhik, A. Jeffrey, Table of integrals, series, and products, 7th ed, Academic Press, Amsterdam; Boston, 2007.

[32] X. Chu, Z. Liu, Comparison between quadratic approximation and $\delta$ expansion in studying the spreading of multi-Gaussian beams in turbulent atmosphere, Appl. Opt. 49 (2010) 204-212.

[33] A. Belafhal, E. El Halba, T. Usman, A closed form of infinite integrals and their application to propagation of Laguerre-Bessel-Gaussian beams, submitted (2020).

[34] A. Belafhal, E. El Halba, T. Usman, Certain integral transforms and their applications in propagation of Laguerre-Gaussian Schell-model beams, Palestine Journal of Mathematics, accepted (2021). 